\title{
VIRTUAL TOURS FOR SMART CITIES: A COMPARATIVE PHOTOGRAMMETRIC APPROACH FOR LOCATING HOT-SPOTS IN SPHERICAL PANORAMAS
}

\author{
R. Feriozzi ${ }^{1}$, A. Meschini ${ }^{1}$, D. Rossi ${ }^{1}$, F. Sicuranza ${ }^{1}$ \\ ${ }^{1}$ University of Camerino, School of Architecture and Design (SAAD), Ascoli Piceno, Italy, (ramona.feriozzi, alessandra.meschini, \\ daniele.rossi, filippo.sicuranza)@unicam.it
}

\section{Commission II}

KEY WORDS: smart city, spherical panoramas, photogrammetry, virtual tour, graphical interfaces

\begin{abstract}
:
The paper aims to investigate the possibilities of using the panorama-based VR to survey data related to that set of activities for planning and management of urban areas, belonging to the Smart Cities strategies. The core of our workflow is to facilitate the visualization of the data produced by the infrastructures of the Smart Cities. A graphical interface based on spherical panoramas, instead of complex three-dimensional could help the user/citizen of the city to better know the operation related to control units spread in the urban area. From a methodological point of view three different kind of spherical panorama acquisition has been tested and compared in order to identify a semi-automatic procedure for locating homologous points on two or more spherical images starting from a point cloud obtained from the same images. The points thus identified allow to quickly identify the same hot-spot on multiple images simultaneously. The comparison shows how all three systems have proved to be useful for the purposes of the research but only one has proved to be reliable from a geometric point of view to identify the locators useful for the construction of the virtual tour.
\end{abstract}

\section{INTRODUCTION}

\subsection{Panorama-based VR}

In recent years the use of immersive technologies developed to support Virtual Reality applications has profoundly changed the way we enjoy graphic information. Most of these technologies, born under the stimulus of the military industry, have matured mainly in the videogame field, thus reaching the general public. VR since its first definition (Lanier, 1992) has been applied in many fields of scientific research. Surgical simulation, architectural previsualization, telepresence and remote collaboration, communication of cultural heritage, education and entertainment are just some of the areas where VR technologies have been tested (Packer and Jordan, 2002).

In particular, this paper aims to investigate the possibilities of using the panorama-based VR to survey data related to that set of activities for planning and management of urban areas, belonging to the Smart Cities strategies.

\subsection{Related works}

In the last decade, Smart cities' have become very popular and for this reason they have been studied in many fields of knowledge. Research has focused in particular on how cities are being instrumented with digital devices and infrastructure that produce 'big data'. Such data enables real-time analysis of city life, as well as new modes of urban governance and smart urbanism (Kitchin, 2014).

Furthermore, in order to implement smart cities, an infrastructure is required to allow the integration of heterogeneous geographical information and sensor networks into a common technological background. In this context, 3D city models became the basis to visualize and manage the modern city information infrastructure. $3 \mathrm{D}$ representations of the urban environment and the possibility to interact with a massive amount of semantic information contained into the geospatial 3D city model has been studied (Prandi et al., 2014). In a similar way 3D GIS city web platform has been elaborated. These platforms are able to load all kinds of demanded data of the city, such as 3D building model data, residents' information, real-time and historical traffic data. These data are then used to conduct graphic analysis and interactive visualizations capable of illustrating the daily activities of the infrastructure networks ( $\mathrm{Li}$ et al., 2015). A further development of the GIS web platforms is the 3D City Web platform based on the Web virtual reality geographical information system. This kind of platform allows the immersive virtual visualization of 3D data represented (Lv et al., 2016).

Regarding the use of spherical panoramas, different applications are designed to navigate both internal spaces and external urban scenarios.

Google Art \& Culture currently allows indoor spaces experiences like virtual visits to many important museums in the world using spherical panoramas both for the exploration of buildings and for browsing their collections. However, different research areas, in connection with museum institutions, have also created specific virtual tour navigations built starting from the acquisition of $360^{\circ}$ panoramic views of the exhibition areas to which are associated contents for interactive consultation both on site through handhelds and for 'visits' remotely. With this solution of virtual exhibits, data in heterogeneous digital archives are integrated according to a semantic model and are enriched by the contribution of domain experts. The scenario is this: a user browsing the panorama selects one of the hot-spot and can visualize, below the panorama, the tabs connected to the selected content. (Mazzoleni et al., 2006; Li-Wei Chan et al., 2005).

In outdoor, panoramic photography, acquired with sophisticated equipment as well as with low-cost fast photogrammetric techniques, is used in many researches for the purposes of 3D reconstructions. In this framework, the image-based mobile mapping systems allow an efficient acquisition of georeferenced image sequences, which can later be exploited in cloud-based 3D geoinformation services. For example, in order to provide a $360^{\circ}$ coverage with accurate 3D measuring capabilities, by using $360^{\circ}$ panorama cameras, were implemented a rigorous sensor and system calibration procedures that allow to rebuild a highly detailed and almost complete 3D city model of the street-level environment (Blaser at al., 2018).

Since long time, some studies deal with developing innovative methods that allow photorealistic $3 \mathrm{D}$ reconstruction with a good level of precision, starting from acquisitions of spherical 
panoramic images integrated and implemented with Structure from Motion techniques and Image-based Modeling.

These methodologies have different purposes such as: musealization of archaeological / architectural heritage through interactive virtual navigation techniques, projections in immersive environments, pseudo holographic representations and WEB GIS publication in open source environment. These applications are oriented both to the fruition from real / virtual visitors and to the management and sharing of information (Fangi et al., 2011).

The wide field of Cultural Heritage include experimentations focused on the creation of Virtual Tours of specific urban areas, ie $2.5 \mathrm{D}$ panoramic views built by joining spherical panoramas acquired on the current urban scene with panoramic views purposefully created from the digital reconstruction of artefacts or architectural projects only imagined, demolished or subsequently transformed. These Virtual Tours aim to offer a contextualized exploration and visualization of the artefact within real urban scenarios to show or 'rebuild' a historical memory.

In this context, and in particular in Italy, specific researches have recently been carried out. Relating to some sites damaged by the latest earthquake in 2016, these researches propose a method of "instant modeling" for the reconstruction of the urban digital model of historical centres. The method underlying the procedures - developed with nodal systems - allows to extract data from the web (an elevation map of the terrain, a map, and spherical panoramas of the historical centre) useful for the model reconstruction. In particular, the "image" data (spherical panoramas from Google Street View) are a very important for rendering the architectural fabric and the appearance of the buildings. These researches aim to reconstruct what have been lost in the earthquake to recover the memory of the places.

Therefore in the Cultural Heritage environment, the use of spherical panoramas representing architectural contexts, conveniently acquired and appropriately integrated with additional contents (historical data of images, textual information, video, audio and other), aim to the construction of interactive virtual tours that can be experienced in web portals equipped of intuitive navigation interfaces (Koeva et al., 2016). Clearly, in this context, the researches concerning smart cities make large use of complicated and less immediate GIS platforms. Indeed the use of these platforms prevails compared to the experimentation of spherical panoramas which, on the contrary, could allow a more simple and intuitive navigation of the related data.

\section{RESEARCH GOAL}

One of the objectives of the research partially presented in this article is to facilitate the analysis of the data produced by the interconnected systems on which the Smart Cities are based, through the use of spherical panoramas, instead of complex threedimensional models.

The aim of the experimentation presented here is to use the panorama-based VR as an interface to access the dynamic data stored by environmental sensors located in urban areas and dedicated to the management of infrastructure networks. This interface could support the traditional consultation methods based on databases referring to GIS systems. A spherical panorama is defined as an image obtained by stitching a series of photographs that share the same point of view. Alternatively, it is possible to acquire a spherical panorama through an omnidirectional camera equipped with at least two wide-angle lenses capable of covering an angle of $360^{\circ}$ in the horizontal plane and $180^{\circ}$ on the vertical axis without interruption.

Both methods of acquisition are now available to everyone considering the relatively low costs of both devices (360 cameras, smartphones, action cameras) and processing software.

Panorama-based VR is often confused with immersive VR. It differs from the latter because it is not possible to move freely within the spherical photo and therefore in a spherical panorama the experience is passive (Xiao, 2000). Similarly, in the VRbased panorama the level of interactivity is very low and is limited only to the selection of some areas of the $360^{\circ}$ image.

These active areas (named hot-spots) on the $360^{\circ}$ image allow both the possibility of linking external content (web pages, texts, pictures), and setting up the so-called Virtual Tour, linking each other's spherical panorama in order to simulate a journey, displaying a sequence of 360 images guided by graphical signals useful to orientate within them (as it happens for Google street view).

The panorama-based VR, as well as virtual tours that exploit the ability to read sequentially images, are based on a technology developed by Apple and dating back to the mid-90s (Quicktime

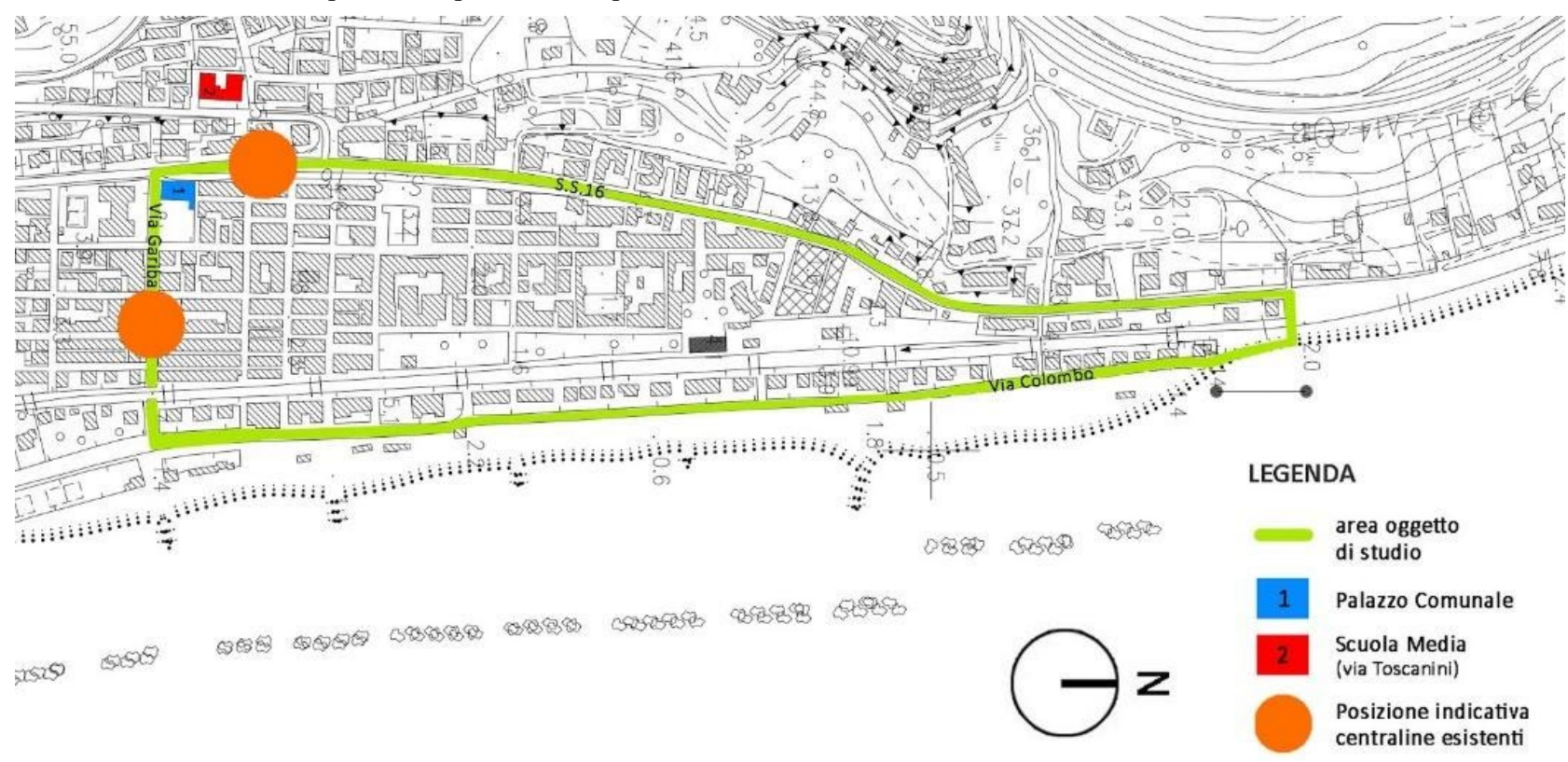

Figure 1: The urban area of Grottammare (Marche, Italy). 
Vr). Since then, many others have been introduced by different developers in the market.

This paper aims to fix a semi-automatic procedure for locating homologous points on two or more spherical images starting from a point cloud obtained from the same images. The points thus identified will allow you to quickly identify the same hotspot on multiple images simultaneously linking on them data from monitoring stations located on the urban area.

\section{CASE STUDY ${ }^{1}$}

The research project involves the implementation of strategies aimed at optimizing and innovating services of a smart city. It has identified as its objective the realization of a prototype of a webbased platform, able to allow, using a geo-referenced 3D mapping base, the integration, management and consultation of a database concerning a series of environmental information (eg air quality, noise pollution, energy consumption related to urban infrastructures, heat loss, etc.) related to specific urban contexts (buildings, roads, service networks and systems, etc.).

The research project focused on an urban area of the territory of the municipality of Grottammare in the Marche region (Italy), classified as a climate zone D. This area has been chosen as a typical example of the widespread Adriatic town and it represents an exemplary urban area that includes all the infrastructural networks (Fig. 1).

The platform integrates the 3D model of the built architectural heritage, obtained through a survey of the area conducted with Mobile Scanner technology, with a geo-database populated by data from the sensor network set up for real-time monitoring.

\section{DEVELOPED METHODOLOGY}

Methodologically, the research has been divided into the following phases:

1. The identification of a smaller urban area to be used as a test for experimentation. For this reason, a part of Via Garibaldi has been chosen. In fact, Via Garibaldi crosses perpendicular both the railway and the Adriatica state road.

It also fits into an urban area characterized by the presence of both residential buildings and hotels as well as other services for the community.

2. The experimentation of different tools and methods of acquisition of spherical panoramas. In particular:

- Samsung Gear 360 spherical camera;

- Nikon D5000 SLR camera with $18 \mathrm{~mm}$ lens mounted on Manfrotto 303SPH panoramic head;

- Equirectangular images used by Google Street View obtained through the use of the svd360.istreetview.com service.

3. Point cloud elaboration, through SFM software (Photoscan), starting from spherical images (Barazzetti, Fangi, Remondino, and Scaioni, 2010).

This operation was carried out with the aim of identifying a spatial relationship between the 3D data and the panoramic images and to indicate, on several images at the same time, a semi-automatic recognition procedure of the position of homologous points in 3D space that correspond to the location of sensor units in urban area (Fig. 2).

${ }^{1}$ The experimentation discussed here is part of research project called I.TM (Innovative Territorial Map) funded under the Programma Operativo Regionale which implements access to funding allocated by the Fondo Europeo di Sviluppo Regionale through the issue of

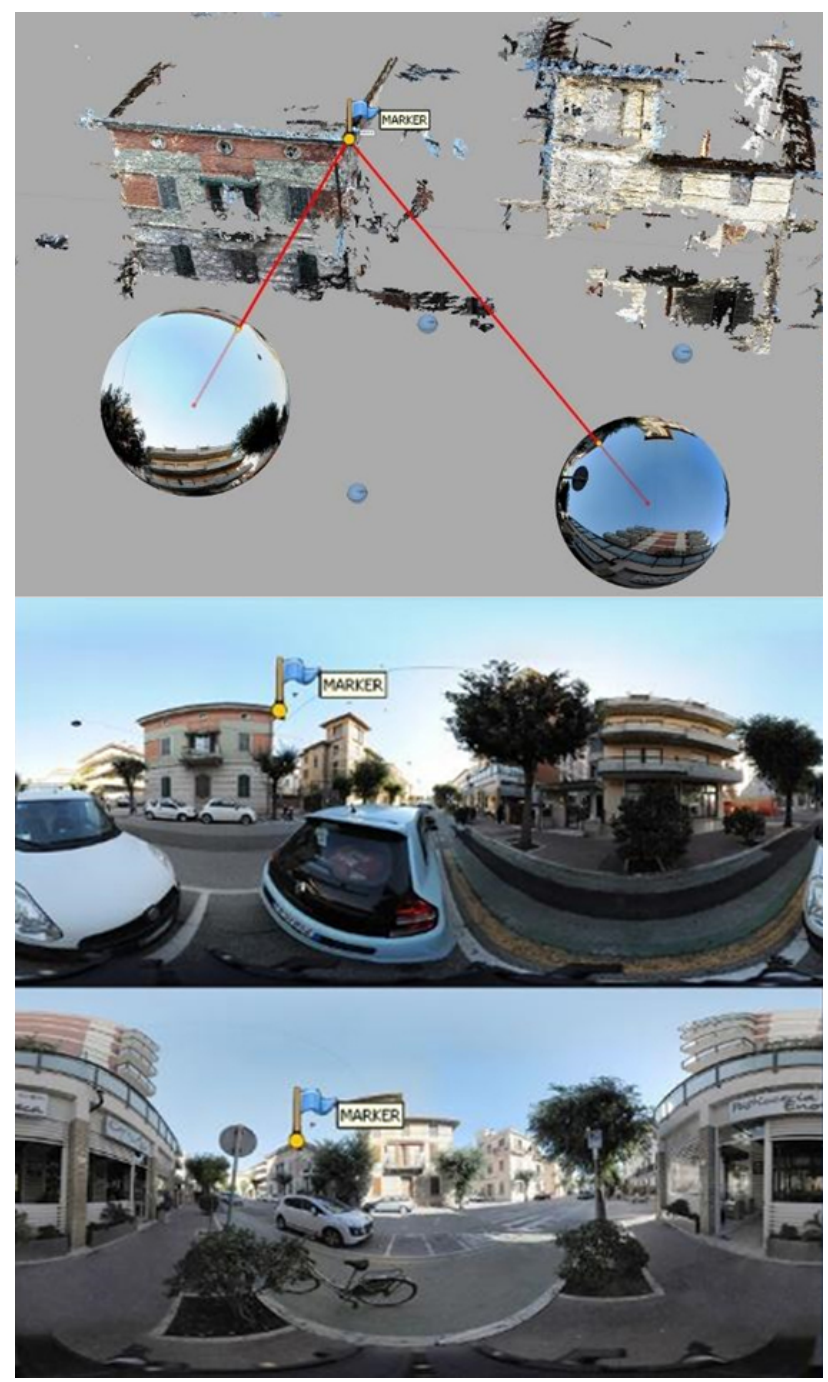

Figure 2: Projection of a marker point from the point of view of a couple of spherical images (top), and the identification of the same marker point on the equirectangular images (bottom).

4. The analysis of the results obtained through the comparison of data. These data come from the technical aspects (instrumentation used, and characteristics of the photographic metadata) and from the timing of shooting campaign (Tab.1). This phase will make it possible to identify instruments and procedures that are closer to achieving the final goal.

5. The design of an interactive virtual tour for the case study area. The virtual tour will be set up with hot-spots to allow the use of additional multimedia content (texts, images, etc.) for real-time monitoring of data from the environmental control units.

\section{DATA COMPARISON}

The comparison between the various techniques made it possible to test their effectiveness both in the first phase of data

specific calls. priority intervention (POR Marche FESR 2014-2020 Asse 1 - azione 1.1 "promozione della ricerca e dello sviluppo negli ambiti della specializzazione intelligente"). 
The International Archives of the Photogrammetry, Remote Sensing and Spatial Information Sciences, Volume XLII-2/W9, 2019 8th Intl. Workshop 3D-ARCH “3D Virtual Reconstruction and Visualization of Complex Architectures", 6-8 February 2019, Bergamo, Italy

\begin{tabular}{|l|l|l|l|l|l|l|}
\hline Tools & $\begin{array}{l}\text { Number of } \\
\text { shooting } \\
\text { locations }\end{array}$ & $\begin{array}{l}\text { Equirectangular } \\
\text { images } \\
\text { resolution }\end{array}$ & $\begin{array}{l}\text { Distance } \\
\text { between the } \\
\text { shooting } \\
\text { locations }\end{array}$ & Shooting time & $\begin{array}{l}\text { Point cloud } \\
\text { resolution }\end{array}$ & $\begin{array}{l}\text { RMS } \\
\text { comparison } \\
\text { error }\end{array}$ \\
\hline $\begin{array}{l}\text { Samsung Gear } \\
\mathbf{3 6 0}\end{array}$ & 38 & $5472 \times 2736$ & $2.5 \mathrm{~m}$ & $\begin{array}{l}5 \mathrm{sec} \text { per } \\
\text { shooting }\end{array}$ & $1 \mathrm{M}$ & $\begin{array}{l}\text { impossible to } \\
\text { determine }\end{array}$ \\
\hline $\begin{array}{l}\text { SLR camera on } \\
\text { panoramic head }\end{array}$ & 11 & $22000 \times 11000$ & $10 \mathrm{~m}$ & $\begin{array}{l}10 \mathrm{~min} \text { per } \\
\text { shooting }\end{array}$ & $2,5 \mathrm{M}$ & $0,38 \mathrm{~m}$ \\
\hline $\begin{array}{l}\text { Equirectangular } \\
\text { images from } \\
\text { Google Street } \\
\text { View }\end{array}$ & 9 & $13312 \times 6656$ & Variable & ND & $2,3 \mathrm{M}$ & $\begin{array}{l}\text { impossible to } \\
\text { determine }\end{array}$ \\
\hline
\end{tabular}

Tab. 1: Comparison of data in relation to the acquisition and processing techniques

acquisition and in subsequent processing and verification of the reliability of the results.

Data in Tab. 1 shows that the resolution of the images significantly influences the number of shooting points and the distance between them because a lower resolution of the images makes it necessary to bring the shooting location closer together and, consequently, increase their number in order to cover the same area of interest.

The data acquired with Samsung Gear 360 show that, despite the high number of stations, the poor resolution of the equirectangular images does not allow to obtain an accurate point cloud (Fangi, Pierdicca, Sturari, Malinverni, 2018).

This result can however be considered satisfactory if we take into account the time-saving shooting campaign (Fig. 3) and the purpose of the study that requires only the positioning of the spherical panoramas into the $3 \mathrm{D}$ space for automatic identification of hotspots.

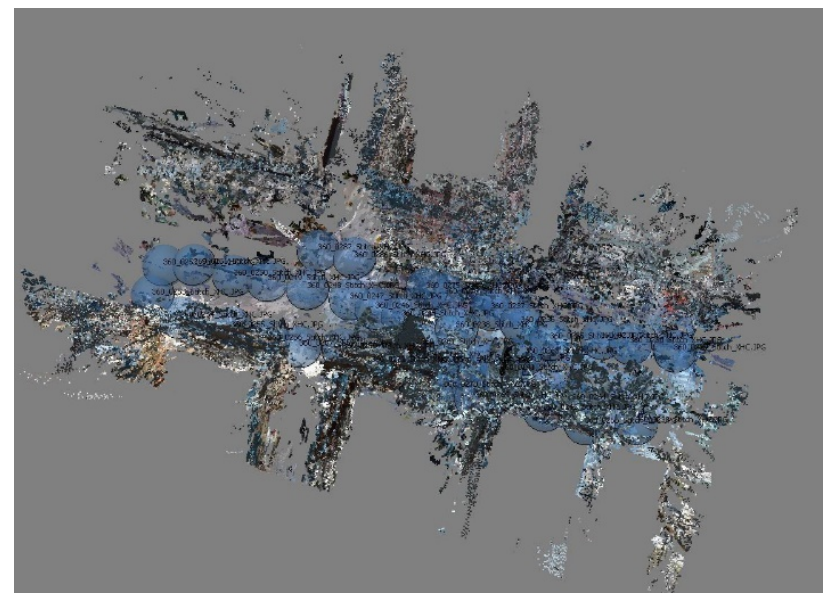

Figure 3: Alignment of the spherical panoramas from Samsung Gear 360 and dense cloud processing.

In the spherical images obtained from SLR camera, the higher resolution of the images resulted in a cloud denser than the previous one but, given the small number of shots, the procedures for processing the data required manual intervention. For this reason and for the long campaign, this procedure is the most complex and time-consuming (Fig. 4).

In addition to the time dedicated to calibration based on the lens and camera used, the photographic campaign presents the common problems related to exposure and focusing. In this case, however, compared to more expeditious technologies, the number of shots that must be adequate to cover the entire sphere of the panorama represents a discriminating factor (for this experimentation, 38 shots were made for each station).

The second processing phase for extrapolation of the point cloud using SFM systems required the preparation of the equirectangular panoramas. For the photos from Samsung Gear the procedure is almost direct, the same cannot be said for the photographs acquired through panoramic head. In fact, these images require software dedicated to stitching such as Kolor Autopano.

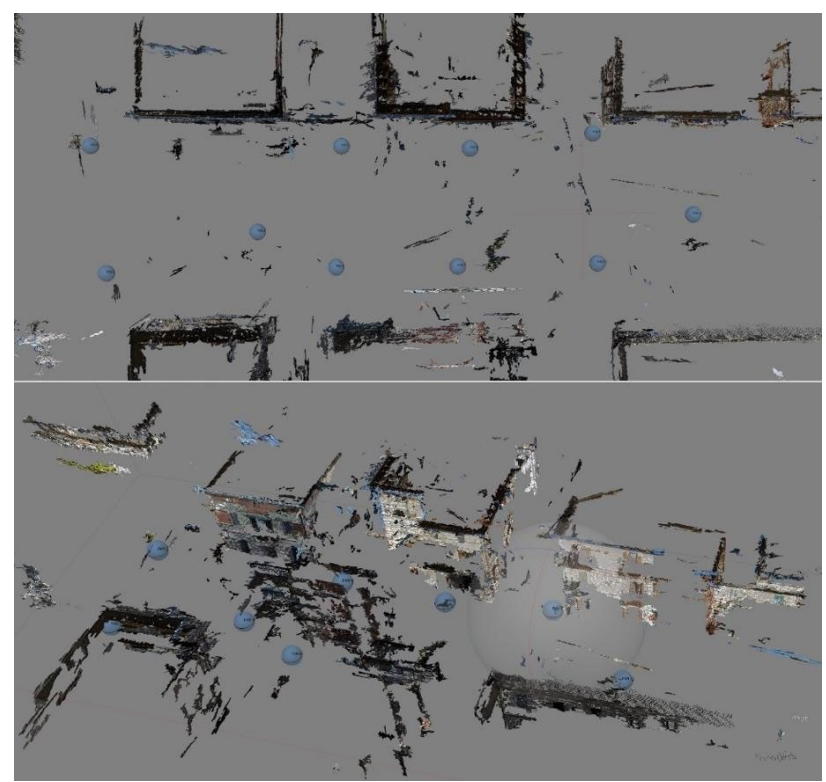

Figure 4: Alignment of the spherical panoramas from SLR camera on panoramic head and dense cloud processing.

Instead, Google Street View photos are a good compromise and they have produced a point cloud almost equivalent to the previous one without the need for a photographic campaign. In this case the issue is the various point cloud distribution with very dense and accurate areas and empty areas.

The biggest difference lies in the different structure of the points cloud. In this case it is possible to notice on one side very dense areas and on the other side completely empty areas (Fig. 5).

In fact, downloading the data already on line has allowed us to avoid the photographic campaign. Street View Download 360 was used for the experimentation. This software allows you to directly download the equirectangular images of the service offered by Google Street View.

Although in this case the acquisition phase is very rapid because it does not require physical presence on the sites, the availability 
of data is not always guaranteed, nor the actual quality of the images. It is not rare to find errors in stitching or graphic artefacts due to the automatic procedures for the composition of the equirectangular projection.

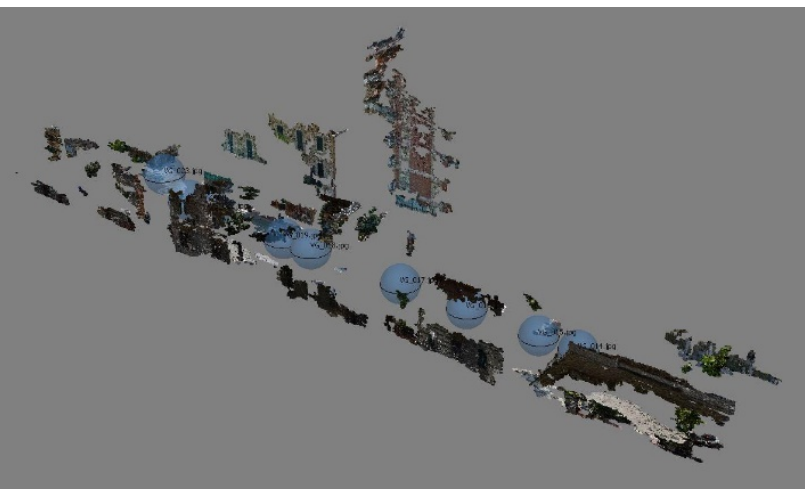

Figure 5: Alignment of the spherical images from Google Street View and dense cloud processing.
The quality and resolution of the point cloud is strictly dependent on the number of equirectangular images available, their resolution, the accuracy of the stitching and the correct exposure of the shots.

These parameters are fully controllable only with the use of Reflex machines on the panoramic head while the 360 cameras do not allow to adjust the exposure or the resolution. With the panoramic images coming from Street View there is obviously less control on the set up of the photographic acquisitions since in this case it is not possible to decide even the number of stations.

For the processing of clouds, we chose to use photogrammetric software such as Photoscan that allows you to process spherical cameras. The experimentation revealed the problems of the software in the phase of alignment of spherical images. These problems are determined to a greater extent by the limited number of panoramas or the poor resolution of the latter.

From these premises, the reliability of the results was evaluated by aligning the three clouds obtained from photogrammetry with that coming from laser scanning that was used as a reference for the calculation of the standard deviation. This parameter, which

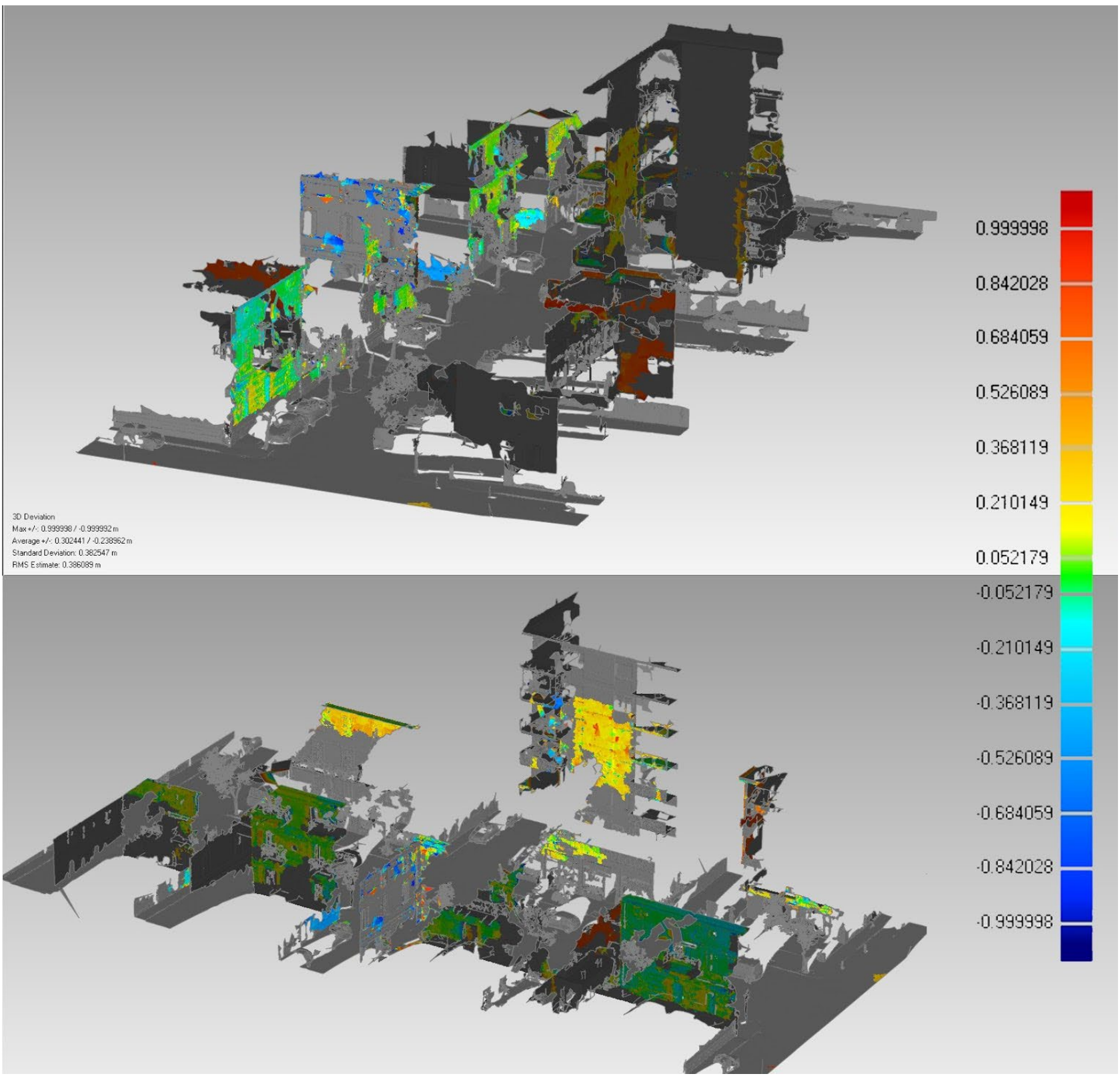

Figure 6: Standard deviation scheme: Point cloud from SLR camera panorama compared to laser scanner point cloud. 
shows the distance of each point of the cloud to be examined with the reference one, allows to quantify the root mean square (RMS) of alignment between the clouds and therefore to mathematically evaluate the accuracy of the data obtained with the three methods.

All three acquisition methods present critical issues:

- Google street view panoramas have good image quality (no noise and stitching artefacts) and a high resolution but are too few for adequate coverage and excessively spaced to allow adequate photogrammetric restitution. Although the RMS of the reprojecting error returned by the bundle adjustment is 0.39 (tie point scale units) and 1.50 (pixel units), the dense cloud coming from the processing has a high number of points (about 2 millions) but concentrated on few areas. For this reason, it is not allowed to identify reference points suitable for alignment with the laser scanner cloud for a verification of the metric error nor the use of the cloud to generate a consistent mesh model.

- Samsung Gear equirectangular images, despite being an adequate number, are the most difficult to process: bad images resolution generates a point cloud with many artifacts that compromise the clarity of results and make the identification of locator point (useful for the alignment of point clouds and calculation of RMS) very difficult.

- The spherical panoramas obtained with a Reflex camera mounted on a panoramic head are certainly the most accurate but it's time consuming and as a result, few photographs were taken since, on sunny days, shots taken a few hours away show substantial differences in lighting making them impossible to process together. The reduced number of shots required a greater effort in the first phase of photomodeling in which manual intervention was necessary. Despite this first obstacle, the final result proved to be satisfactory. In this case the alignment with the reference cloud allowed to perform the analysis of the deviation and to highlight the root mean square which proved to be absolutely negligible for the purposes of experimentation (Fig. $6)$.

In short, the three methods proved adequate for the semiautomatic detection of hotspots.

This detection in the cases of images coming from Google Street View and Gear 360 requires the location of points only through photos, while in the case of spherical panoramas derived from the SRL camera the points can be placed either by using only the photos, or through the placement of markers on the dense point cloud or on the derived mesh.

In fact, if the alignment of the panoramas is sufficient in all three cases, instead it is not sufficient the distribution of points of the dense cloud, with large gaps in that resulting from the spherical views of Google Street View and with numerous artefacts in that from Samsung Gear.

\section{CONCLUSIONS}

In this paper we have presented a comparison between different method to acquire spherical panorama in order to visualize and interact in real time with smart cities. Spherical panoramas can be translated in graphical interfaces to access data characterizing smart cities coming from a series of sensors located in urban areas.

This type of visualization arises as an alternative to the traditional way of fruition using a GIS map in order to facilitate the way of browsing complex data in the perspective of a 'smart' social participation in the management of the city.
The studies conducted for the realization of this objective have focused in particular on the attempt to design an interface based on an immersive and continuous vision of the study area starting from the acquisition and use of spherical panoramas.

In other words, setting up a 'virtual tour' to allow a smooth exploration of the urban area according to a mode closer to real perception.

However, it should be noted that it was not a photographic campaign aimed at a geometrical survey of 3D space but rather a photographic acquisition that aimed essentially at allowing an interactive navigation of space through the alignment of an appropriate sequence of spherical panoramas and the predisposition of hotspots for the query of heterogeneous environmental data detected by sensors located at strategic points of the study area.

The spherical panoramas examined were obtained in three different ways and they were processed in order to identify a semi-automatic procedure for locating homologous points on two or more spherical images starting from a point cloud obtained from the same images.

The points thus identified allowed us to quickly identify the same hot-spot on multiple images simultaneously helping in designing the interaction between the user/citizen and the data coming from the management of smart cities infrastructures.

\section{ACKNOWLEDGEMENTS}

The authors would like to acknowledge Tecno Art s.r.l. for providing the scanner laser point cloud.

\section{REFERENCES}

Lanier, J., 1992. Virtual reality: The promise of the future. Interactive Learning International, 8(4), 275-79.

Yi Xiao, D., 2000. Experiencing the library in a panorama virtual reality environment. Library Hi Tech, 18(2), 177-184.

Packer, R. and Jordan, K. (Eds.), 2002. Multimedia: from Wagner to virtual reality. WW Norton \& Company.

Chan L.-W., Hsu Y.-Y., Hung Y.-P. and Hsu J. Y.-J., 2005. Orientation-Aware Handhelds for Panorama-Based Museum Guiding System. In: UbiComp 2005 Workshop: Smart Environments and their Applications to Cultural Heritage, pp. 43-46.

Mazzoleni P., Valtolina S., Franzoni S., Mussio P. and Bertino E., 2006. Towards a contextualized access to the cultural heritage world using 360 Panoramic Images. In: 18th International Conference on Software Engineering and Knowledge Engineering (SEKE 2006), pp. 416-419.

Barazzetti, L., Fangi, G., Remondino, F. and Scaioni, M., 2010. Automation in multi-image spherical photogrammetry for $3 \mathrm{~d}$ architectural reconstructions. VAST2010, pp. 1-6.

Capotorto S., Maiellaro N. and Zonno M., 2010. Ambienti immersivi geolocalizzati per la fruizione a distanza di beni culturali. In: Atti $14^{a}$ Conferenza Nazionale ASITA, pp. 457-462.

Fangi G., Malinverni E.S., D’Annibale E., Tassetti A.N. and Bozzi C.A., 2011. Metodi innovativi per la documentazione e valorizzazione di siti archeologici. In: Atti $15^{\circ}$ Conferenza Nazionale ASITA, pp. 981-992.

Ippoliti E., Calvano M. and Mores L., 2014. 2.5D/3D models for the enhancement of architectural-urban heritage. A virtual tour of design of the fascist headquarters in Littoria. ISPRS Annals of the 
Photogrammetry, Remote Sensing and Spatial Information Sciences, vol. II-5, pp. 189-196.

Kitchin, R., 2014. The real-time city? Big data and smart urbanism. GeoJournal, vol. 79(1), pp. 1-14.

Prandi, F., Soave, M., Devigili, F., Andreolli, M. and De Amicis, R., 2014. Services oriented smart city platform based on 3D city model visualization. ISPRS Annals of the Photogrammetry, Remote Sensing and Spatial Information Sciences, vol. 2(4), pp.59-64.

Li, X., Lv, Z., Hu, J., Zhang, B., Shi, L. and Feng, S., 2015. XEarth: A 3D GIS Platform for managing massive city information. In: Computational Intelligence and Virtual Environments for Measurement Systems and Applications (CIVEMSA), 2015 IEEE International Conference on, pp. 1-6.

Lv, Z., Li, X., Zhang, B., Wang, W., Zhu, Y., Hu, J. and Feng, S., 2016. Managing big city information based on WebVRGIS. IEEE Access, 4, pp. 407-415.

Calvano M. and Casale A., 2017. Il modello integrato e la conservazione dell'immagine della città. In: 17th CIRIAF National Congress. Sustainable Development, Human Health and Environmental Protection, pp. 1-13.

Koeva M., Luleva M. and Maldjanski P., 2017. Integrating Spherical Panoramas and Maps for Visualization of Cultural Heritage Objects Using Virtual Reality Technology. Sensors vol. 17 , issue 829 , pp. 1-15.

Blaser S., Nebiker S. and Cavegn S., 2018. On a Novel $360^{\circ}$ Panoramic Stereo Mobile Mapping System. Photogrammetric Engineering and Remote Sensing vol. 84(6), pp. 347-356.

Fangi, G., Pierdicca, R., Sturari, M. and Malinverni, E.S., 2018. Improving Spherical Photgrammetry Using $360^{\circ}$ OmniCameras: Use Cases and New Spplications. ISPRS - The International Archives of the Photogrammetry, Remote Sensing and Spatial Information Sciences. Volume XLII-2, 331-337. 